\title{
Estudo de suspensões de bentonitas sob diferentes condições térmicas
}

\section{(Study of bentonit suspensions under different thermal conditions)}

\author{
C.A. Leal ${ }^{1}$, R.C.A.M.Nascimento ${ }^{2}$, L. V.Amorim ${ }^{3}$ \\ ${ }^{1}$ Unidade Acadêmica de Engenharia de Materiais, UFCG, \\ ${ }^{2} P P G C E M a t, C C T, U F C G$ \\ ${ }^{3} U A E M, C C T, U F C G$ \\ Rua Aprígio Veloso 882, Campina Grande, PB 58429-900 \\ luciana@dem.ufcg.edu.br
}

\begin{abstract}
Resumo
Suspensões argilosas são utilizadas como fluidos de perfuração de poços nas primeiras camadas rochosas, geralmente constituídas por sedimentos inconsolidados. Em camadas mais profundas são comumente utilizados fluidos base óleo devido sua resistência térmica. No entanto, em virtude das leis ambientais estes fluidos estão sendo substituídos e as suspensões argilosas surgem como uma alternativa pelo caráter ambientalmente correto e menor custo. Assim, torna-se de extrema importância o estudo das propriedades de suspensões argilosas a temperaturas moderadas e elevadas. Desta forma, este trabalho tem por objetivo avaliar as propriedades das suspensões argilosas de bentonita sob diferentes condições térmicas. Para tanto, foi empregado um planejamento fatorial do tipo $2^{2}$ com três experimentos no ponto central + configuração estrela, totalizando 11 corridas experimentais para avaliar a influência da temperatura e do teor de argila (variáveis de entrada) sobre as propriedades reológicas e de filtração das suspensões argilosas. As suspensões foram preparadas com concentração de bentonita variando de $8,75 \mathrm{~g} / 350 \mathrm{~mL}$ a $22,4 \mathrm{~g} / 350 \mathrm{~mL}$ e submetidas a temperaturas entre $38{ }^{\circ} \mathrm{C}$ e $176^{\circ} \mathrm{C}$ durante $16 \mathrm{~h}$. Foram estudados dois tipos de envelhecimento térmico, dinâmico e estático, em equipamento Roller Oven da Fann. As propriedades reológicas (viscosidades aparente - VA e plástica - VP) foram determinadas em viscosímetro Fann 35A e o volume de filtrado (VF) em filtro-prensa API. Concluiu-se que as variáveis de entrada influenciam estatisticamente, ao nível de $95 \%$ de confiança, as propriedades das dispersões, sendo observado que a combinação de temperaturas elevadas e altas concentrações de argila conduz a maiores valores de VA e VP, bem como que os maiores valores de VF foram obtidos com as suspensões preparadas com baixa concentração de argila e submetidas à elevada temperatura. Ficou evidenciado ainda que o tipo de envelhecimento tem influência direta no comportamento reológico das dispersões avaliadas.
\end{abstract}

Palavras-chave: suspensões argilosas, bentonita, envelhecimento, estabilidade térmica.

\begin{abstract}
Clay suspensions are used as drilling fluids for the initial rock layers, usually constituted of unconsolidated sediments. For deeper layers oil based fluids are more commonly used due to their thermal resistance. Therefore, because of the environmental laws, these fluids are being replaced and the clay suspensions arise as an alternative, as they are environmentally correct and have lower cost. So, it becomes of great importance to study the properties of clay suspensions at moderate and high temperatures. This work has the aim to evaluate the properties of bentonit clay suspensions under different thermal conditions. For so, a type $2^{2}$ factorial plan with three experiments in the centre + star configuration, was applied, totalizing 11 experimental runs to evaluate the influence of temperature and clay content (entrance variables) over the rheological and filtration properties of the clay suspensions. The clay suspensions were prepared with bentonit concentrations between $8.75 \mathrm{~g} / 350 \mathrm{~mL}$ and $22.4 \mathrm{~g} / 350 \mathrm{~mL}$ and were submitted to temperatures between $38{ }^{\circ} \mathrm{C}$ and $176{ }^{\circ} \mathrm{C}$ during $16 \mathrm{~h}$. Two types of thermal aging were studied, dynamic and static, in a Fann Roller Oven equipment. The rheological properties (apparent viscosity - AV and plastic viscosity - PV) were determined in a Fann $35 A$ viscometer and the filtered volume (FV) in a API filter-press. Could be concluded that the entrance variables did influence statistically, at a 95\% confidence level, on the dispersion properties, being observed that the combination of elevated temperatures and high clay concentrations leads to higher values of VA and VP, as for the higher values of VF were obtained from the suspensions prepared with lower clay concentrations and submitted to higher temperature. It became evident that the type of aging does have direct influence in the rheological behaviors of the evaluated dispersions.
\end{abstract}

Keywords: clay suspensions, bentonite, aging, thermal stability.

\section{INTRODUÇÃO}

Os sistemas coloidais são sistemas em que a fase dispersa é geralmente um sólido ou um liquido e a fase dispersante é geralmente um líquido, sendo água o caso mais freqüente.
As dispersões coloidais são constituídas por partículas entre $0,1 \mu \mathrm{m}$ e $0,001 \mu \mathrm{m}$ [1]. Dispersões coloidais diluídas de bentonitas sódicas com certo grau de defloculação apresentam o fenômeno de tixotropia. A suspensão torna-se menos viscosa pela agitação e mais espessa pelo repouso. 
Essa propriedade é de extrema importância para a aplicação dessas suspensões como fluidos de perfuração de poços de petróleo. A tixotropia tende a aumentar com a presença de partículas de cargas opostas que pode formar pontes de ligações, assim como também sofre alterações com o tempo (envelhecimento) [1].

As partículas de argila, na forma dispersa, podem associar-se de três formas diferentes: face-a-face, facea-aresta e aresta-a-aresta [2]. A agregação ocorre em função da associação do tipo face-a-face, conduzindo à formação de plaquetas, diminuindo assim, o numero de partículas e reduzindo a viscosidade. Já a floculação ocorre em função das associações aresta-aresta e/ou aresta-face das partículas, conduzindo à formação de uma estrutura "castelo de cartas", aumentando a viscosidade [3]. A associação de partículas determina o comportamento reológico de suspensões de bentonita, sendo de grande importância aos fluidos de perfuração [2]. A bentonita é a argila comercial mais utilizada em fluidos à base de água doce, sendo adicionada para desempenhar uma ou várias das seguintes funções: aumentar a capacidade de limpeza do poço, reduzir as infiltrações nas formações permeáveis, formar uma membrana de baixa permeabilidade (reboco), promover a estabilidade do poço e evitar ou superar a perda de circulação [4]. As suspensões argilosas são geralmente empregadas nas primeiras fases da perfuração de poços, compostas geralmente por sedimentos inconsolidados [5]. Nas fases posteriores, onde os poços atingem grandes profundidades, é comum a presença de formações geológicas de difícil perfuração, a exemplo de folhelhos expansivos, bem como condições de elevadas temperaturas. Quando a perfuração atinge, portanto, estas fases, os fluidos permanecem expostos às condições presentes por longos períodos de tempo, ainda mais quando da necessidade de operações rotineiras como as manobras, nas quais é retirada toda a coluna de perfuração para substituição da broca. As suspensões argilosas são sensíveis a altas temperaturas e quando expostas a estas condições por longos períodos formam géis rígidos, podendo comprometer as operações de perfuração. No passado, para superar esses problemas, eram utilizados os fluidos à base de óleo diesel, pois esses permaneciam estáveis a temperaturas elevadas. No entanto, devido a considerações ambientais, logísticos e aos altos custos, o uso desse tipo de fluido tornou-se inviável [6].

As propriedades reológicas dos fluidos de perfuração podem apresentar grandes diferenças a temperatura ambiente e no interior do poço [7]. A temperatura no interior do poço depende do gradiente geotérmico e pode ser superior a $260{ }^{\circ} \mathrm{C}$. Mesmo as temperaturas moderadas podem ter influência signifiticativa nas propriedades reológicas dos fluidos. O fluido no interior do poço pode ser mais espesso ou mais fino do que na superfície, e um aditivo que reduz a viscosidade na superfície pode aumentar a viscosidade do fluido no interior do poço. A reologia dos fluidos base água com bentonita em alta temperatura foi estudada [8]. Os resultados mostraram que se a suspensão é totalmente defloculada, a viscosidade plástica e o limite de escoamento diminuem com o aumento da temperatura até $177^{\circ} \mathrm{C}$, enquanto que se a suspensão for floculada, apenas a viscosidade plástica diminui e o limite de escoamento aumenta drasticamente em temperaturas superiores a $100{ }^{\circ} \mathrm{C}$. Vários estudos realizados indicam que o efeito da temperatura sobre as propriedades reológicas dos fluidos de perfuração aditivados com bentonita é imprevisível, sendo asssim os mecanimos de ação não são totalmente compreendidos [6-13]. Com isso, este trabalho tem como objetivo avaliar as propriedades das suspensões argilosas de bentonita sódica submetidas ao envelhecimento sob diferentes condições térmicas.

\section{MATERIAIS E MÉTODOS}

Foi estudada uma amostra de argila bentonítica sódica industrializada em Campina Grande (PB), proveniente das jazidas localizadas no município de Boa Vista, PB, cedida pela Empresa Bentonit União Nordeste e comercialmente conhecida por Brasgel PA. Essa argila é amplamente utilizada na indústria de petróleo como agente viscosificante, tixotrópico e formador de reboco.

\section{Metodologia}

\section{Planejamento Fatorial}

Para avaliar a influência das variáveis de entrada (concentração de argila e temperatura) sobre as propriedades reológicas (viscosidades aparente e plástica) e de filtração (volume de filtrado) das suspensões argilosas, foi utilizado um planejamento fatorial do tipo $2^{2}$ com três experimentos no ponto central + configuração estrela, totalizando 11 corridas experimentais [14]. Os experimentos foram realizados em duas condições de envelhecimento. Assim, foram desenvolvidos dois planejamentos, um na condição de envelhecimento estático e outro na condição de envelhecimento dinâmico. É importante ressaltar que inicialmente foi utilizado o planejamento fatorial do tipo $2^{2}$ com três experimentos no ponto central, contudo o modelo linear obtido não apresentou resultados satisfatórios. Desta forma, utilizou-se um modelo quadrático por meio da ampliação do planejamento, ou seja, fazendo-se uso do planejamento com configuração estrela [14]. Para tanto, foi acrescentado ao planejamento original um outro idêntico, porém girado de $45^{\circ}$ em relação à orientação de partida. A regressão dos dados experimentais foi realizada com o software Statistica, versão 7.0 [15]. Os valores codificados e reais das variáveis de entrada empregados no planejamento encontram-se na Tabela I e na Tabela II está apresentada a matriz de planejamento estudada.

\section{Preparação das suspensões argilosas}

A argila foi adicionada nas concentrações de $8,75 \mathrm{~g} /$ $350 \mathrm{~mL}, 10,79 \mathrm{~g} / 350 \mathrm{~mL}, 15,58 \mathrm{~g} / 350 \mathrm{~mL}, 20,37 \mathrm{~g} / 350 \mathrm{~mL}$ e $22,4 \mathrm{~g} / 350 \mathrm{~mL}$ sob agitação constante a uma velocidade 
Tabela I - Valores codificados e reais das variáveis de entrada empregados no planejamento fatorial. [Table I - Coded and real values of input variables used in the factorial design.]

\begin{tabular}{cccccc}
\hline & \multicolumn{5}{c}{ Níveis codificados } \\
Variáveis de entrada & Nível & Nível & Ponto central & Nível & Nível \\
& $-\infty$ & -1 & 0 & +1 & $+\infty$ \\
\hline Concentração de argila $(\mathrm{g} / 350 \mathrm{~mL})$ & 8,75 & 10,79 & 15,58 & 20,37 & 22,4 \\
Temperatura de envelhecimento $\left({ }^{\circ} \mathrm{C}\right)$ & 38 & 58 & 107 & 156 & 176 \\
\hline
\end{tabular}

Tabela II - Matriz de planejamento experimental. [Table II - Matrix of experimental design.]

\begin{tabular}{ccc}
\hline $\begin{array}{c}\text { Experimentos } \\
\text { (Suspensões) }\end{array}$ & $\begin{array}{c}\text { Concentração de } \\
\text { argila }(\mathrm{g} / 350 \mathrm{~mL})\end{array}$ & $\begin{array}{c}\text { Temperatura de } \\
\text { envelhecimento } \\
\left({ }^{\circ} \mathrm{C}\right)\end{array}$ \\
\hline 1 & $-1(10,79)$ & $-1(58,0)$ \\
2 & $+1(20,37)$ & $-1(58,0)$ \\
3 & $-1(10,79)$ & $+1(156,0)$ \\
4 & $+1(20,37)$ & $+1(156,0)$ \\
5 & $-\infty(8,75)$ & $0(107,0)$ \\
6 & $+\infty(22,4)$ & $0(107,0)$ \\
7 & $0(15,58)$ & $-\infty(38,0)$ \\
8 & $0(15,58)$ & $+\infty(176,0)$ \\
9 & $0(15,58)$ & $0(107,0)$ \\
10 & $0(15,58)$ & $0(107,0)$ \\
11 & $0(15,58)$ & $0(107,0)$ \\
\hline
\end{tabular}

de $13.000 \mathrm{rpm}$ em agitador Hamilton Beach 936. Após adição da argila, a velocidade do agitador foi aumentada para $17.000 \mathrm{rpm}$, permanecendo por 20 min sob agitação constante.

\section{Envelhecimento das suspensões argilosas}

Após a preparação das suspensões, as mesmas foram submetidas ao envelhecimento em forno Roller Oven 705 ES Fann nas seguintes temperaturas: $38{ }^{\circ} \mathrm{C}, 58{ }^{\circ} \mathrm{C}, 107^{\circ} \mathrm{C}$, $156{ }^{\circ} \mathrm{C}$ e $176{ }^{\circ} \mathrm{C}$. O envelhecimento foi realizado tanto em condições estáticas como em condições dinâmicas durante $16 \mathrm{~h}$. Em condição estática, o fluido acondicionado em células de aço inox permanece em repouso no interior do equipamento Roller Oven, enquanto que em condição dinâmica, o fluido acondicionado de forma semelhante rola sobre rolos no interior deste equipamento.

\section{Estudo reológico}

Para o estudo reológico, a suspensão foi agitada durante 5 min em agitador mecânico Hamilton Beach 936 a $17.000 \mathrm{rpm}$. Em seguida, a suspensão foi transferida para o recipiente do viscosímetro Fann 35A. O equipamento foi acionado a $600 \mathrm{rpm} / 2 \mathrm{~min}$ e efetuada a leitura. Em seguida, a velocidade foi mudada para $300 \mathrm{rpm}$ e efetuada a leitura após $15 \mathrm{~s}$. As viscosidades aparente e plástica foram obtidas segundo a norma EP-1EP-00011-A [16]. A viscosidade aparente (VA) é o valor obtido na leitura a $600 \mathrm{rpm}$ dividido por 2, dada em cP. A viscosidade plástica (VP) é a diferença das leituras realizadas a $600 \mathrm{rpm}$ e a $300 \mathrm{rpm}$, dada também em cP.

\section{Volume de filtrado}

Após a determinação dos parâmetros reológicos foi determinado o volume de filtrado (VF). Para tanto, as suspensões foram agitadas durante $1 \mathrm{~min}$, em agitador mecânico Hamilton Beach 936 a 17.000 rpm. Em seguida, foram transferidas para os recipientes do filtro-prensa API e submetidas a uma pressão da ordem de 100 psi. Após 30 min, o filtrado foi lido e obteve-se a medida do volume do filtrado, expresso em $\mathrm{mL}$, de acordo com a norma EP-1EP00011-A [16].

\section{RESULTADOS E DISCUSSÃO}

Nas suspensões argilosas, o teor de argila tem bastante influência nas propriedades reológicas e de filtração. Além disto, conforme mencionado, estas suspensões são comumente utilizadas como fluidos de perfuração nas primeiras fases da perfuração de poços [5]. Devido ao grande número de variáveis envolvidas, o comportamento dos fluidos de perfuração em altas temperaturas, particularmente os fluidos base água, a exemplo das dispersões de bentonitas sódicas, é imprevisível e, de fato, ainda não totalmente compreendido. Variações muito pequenas na composição destes fluidos podem fazer diferenças consideráveis no seu comportamento reológico, de modo que é necessário testar cada fluido individualmente, a fim de obter dados confiáveis [17]. O comportamento reológico e de filtração das suspensões argilosas foram avaliados em relação às variáveis concentração de argila e temperatura, tanto em condições de envelhecimento estático como dinâmico, conforme apresentado no item anterior. Desta forma, na Tabela III estão apresentados os resultados das propriedades reológicas (viscosidade aparente e plástica) e de filtração (volume de filtrado) das suspensões argilosas.

Em todas as suspensões argilosas estudadas o aumento da temperatura no nível $(+1)$ resultou em maiores valores de viscosidades aparente e plástica e de volume de filtrado. Para as suspensões com teores mais elevados de argila (S2, temperatura no nível -1 e S4, temperatura no nível +1 ) submetidos a condições estáticas, o aumento 
da viscosidade aparente $\left(\mathrm{VA}_{\mathrm{E}}\right)$ foi de $80,95 \%$. Para as mesmas suspensões, sendo que submetidas a condições dinâmicas, o aumento da viscosidade aparente $\left(\mathrm{VA}_{\mathrm{D}}\right)$ foi de apenas 2,74\%. Provavelmente, esta pequena variação da viscosidade, deve-se a melhor dispersão da argila na água, pois durante o envelhecimento dinâmico, a suspensão, acondicionada numa célula de. aço inox, gira sobre rolos, permanecendo em constante movimento durante todo período de envelhecimento que é de $16 \mathrm{~h}$. Como esperado, as suspensões com maiores teores de argila apresentaram maiores valores de VA e de VP. O aumento nos valores dessas propriedades decorre da maior intensidade das interações eletrostáticas e de massa entre as partículas sólidas, que se tornam predominantes com o aumento da concentração de argila [2]. Comparando as suspensões S5, S6, S9, S10 e S11, que foram submetidas ao envelhecimento na mesma temperatura, variando apenas o teor de argila, foi possível observar que a suspensão S6 apresentou o maior valor de VA, para ambas as condições de envelhecimento, sendo a suspensão submetida ao envelhecimento dinâmico, a que apresentou o maior valor de $\mathrm{VA}_{\mathrm{D}}, 67,5 \mathrm{cP}$. Quanto a VP, definida como uma variável dependente da interação dos sólidos presentes e/ou uma medida da fricção resultante do choque de uma partícula com outra [18], a suspensão S6 submetida ao envelhecimento estático apresentou valor de $14,0 \mathrm{cP}\left(\mathrm{VP}_{\mathrm{E}}\right)$. As suspensões submetidas ao envelhecimento dinâmico (S4) apresentaram valor próximo a 16,0 cP. Em ambas as condições de envelhecimento, o aumento da concentração de argila de $10,79 \mathrm{~g} / 350 \mathrm{~mL}$ para $20,37 \mathrm{~g} /$ $350 \mathrm{~mL}$ (aumento de $88,79 \%$ ) resultou em um efeito positivo para o VF, sendo observado uma redução de $43,26 \%$, na condição estática e 34,55\%, na condição dinâmica.

Por meio da análise da Tabela III, as suspensões S2, S4 e S6, em ambas as condições de envelhecimento (estático e dinâmico), apresentam-se em sistemas denominados floculado-gel, traduzido pelos elevados valores de VA e VF e baixos valores de VP, sendo as suspensões submetidas ao envelhecimento dinâmico, as que apresentaram um maior grau de floculado-gel. Neste estado, as partículas de argila permanecem unidas através de interações elétricas e de massa, com associações do tipo face-a-aresta e arestaa-aresta, gerando uma estrutura do tipo castelo-de-cartas, na qual, a fase líquida encontra-se presa no interior dessa estrutura e/ou livre [1]. Quando são obtidos valores de VF elevados, bem como altos valores de VA e baixos valores de VP, tem-se o caso de uma suspensão no estado floculadogel com a fase líquida livre, que aparece sobrenadante na suspensão.

Nas Tabelas IV e V estão apresentadas as análises de variância e os modelos matemáticos codificados para as propriedades reológicas (VA e VP) e para o volume de filtrado (VF) das suspensões argilosas submetidas ao envelhecimento estático e dinâmico, respectivamente.

Por meio da análise das Tabelas IV e $\mathrm{V}$ foi possível perceber que nos dois tipos de envelhecimento (estático e dinâmico) a razão entre o $\mathrm{F}_{\text {calculado }}$ e $\mathrm{F}_{\text {tabelado }}$, para todas as respostas (VA, VP e VF), apresentaram valores superiores a 5, obtendo assim modelos matemáticos estatisticamente significativos e preditivos. Também foi observado, através da razão entre $o F_{\text {calculado }}$ e $o F_{\text {tabelado }}$, para o erro puro, que na maior parte das respostas o modelo não necessita de ajuste. Nos casos estudados, a análise de significância estatística mostrou que os coeficientes de correlação (R) e os coeficientes de variação explicada $\left(\mathrm{R}^{2}\right)$ dos resultados experimentais para VA, VP e VF das suspensões foram satisfatórios, superiores a 0,977 e $97,0 \%$ (Tabela IV) e 0,977 e 95,439\% (Tabela V), respectivamente. Deste modo, pode-se dizer que os modelos matemáticos apresentados nas Tabelas IV e V têm mais de 97,0\% (Tabela IV) e 95,439\% (Tabela V) das variações obtidas explicadas pelo modelo. Para o envelhecimento estático, a análise dos efeitos principais, a interação entre eles e as variáveis quadráticas para as viscosidades aparente

Tabela III - Propriedades reológicas e de filtração das suspensões argilosas.

[Table III - Rheological and filtration of clay suspensions.]

\begin{tabular}{ccccccccc}
\hline $\begin{array}{c}\text { Experimentos } \\
\text { (Suspensões) }\end{array}$ & $\begin{array}{c}\text { Concentração de } \\
\text { argila }(\mathrm{g} / 350 \mathrm{~mL})\end{array}$ & $\begin{array}{c}\text { Temperatura de } \\
\text { envelhecimento }\left({ }^{\circ} \mathrm{C}\right)\end{array}$ & $\begin{array}{c}\mathrm{VA}_{\mathrm{E}} \\
(\mathrm{cP})\end{array}$ & $\begin{array}{c}\mathrm{VA}_{\mathrm{D}} \\
(\mathrm{cP})\end{array}$ & $\begin{array}{c}\mathrm{VP}_{\mathrm{E}} \\
(\mathrm{cP})\end{array}$ & $\begin{array}{c}\mathrm{VP}_{\mathrm{D}} \\
(\mathrm{cP})\end{array}$ & $\begin{array}{c}\mathrm{VF}_{\mathrm{E}} \\
(\mathrm{mL})\end{array}$ & $\begin{array}{c}\mathrm{VF}_{\mathrm{D}} \\
(\mathrm{mL})\end{array}$ \\
\hline $1(\mathrm{~S} 1)$ & -1 & -1 & 6,0 & 8,5 & 4,0 & 5,0 & 28,2 & 27,5 \\
$2(\mathrm{~S} 2)$ & +1 & -1 & 21,0 & 36,5 & 9,0 & 5,0 & 16,0 & 18,0 \\
$3(\mathrm{~S} 3)$ & -1 & +1 & 9,0 & 12,0 & 6,5 & 3,0 & 38,6 & 35,8 \\
$4(\mathrm{~S} 4)$ & +1 & +1 & 38,0 & 37,5 & 14,0 & 16,0 & 23,0 & 24,0 \\
$5(\mathrm{~S} 5)$ & $-\infty$ & 0 & 6,5 & 6,5 & 5,0 & 4,5 & 33,4 & 34,4 \\
$6(\mathrm{~S} 6)$ & $+\infty$ & 0 & 36,7 & 67,5 & 14,5 & 11,0 & 17,6 & 18,6 \\
$7(\mathrm{~S} 7)$ & 0 & $-\infty$ & 12,3 & 13,5 & 7,0 & 7,0 & 19,8 & 20,0 \\
$8(\mathrm{~S} 8)$ & 0 & $+\infty$ & 16,8 & 16,0 & 11,5 & 11,0 & 28,4 & 31,4 \\
$9(\mathrm{~S} 9)$ & 0 & 0 & 18,7 & 26,8 & 9,5 & 10,5 & 24,0 & 23,6 \\
$10(\mathrm{~S} 10)$ & 0 & 0 & 19,5 & 27,0 & 10,0 & 11,0 & 23,8 & 24,8 \\
$11(\mathrm{~S} 10)$ & 0 & 0 & 18,7 & 26,5 & 9,5 & 11,0 & 23,4 & 24,0 \\
\hline
\end{tabular}

$V A_{E}, V P_{E}$ e $V F_{E}$ - viscosidade aparente, viscosidade plástica e volume de filtrado na condição de envelhecimento estático e $V A_{D}, V P_{D}$ e $V F_{D}$ - viscosidade aparente, viscosidade plástica e volume de filtrado na condição de envelhecimento dinâmico. 
Tabela IV - Análises de variância (ANOVA) e modelos matemáticos codificados das propriedades reológicas e de filtração das suspensões argilosas para o planejamento fatorial empregado sob envelhecimento estático.

[Table IV - Analysis of variance (ANOVA) and encoded mathematical models of the rheological properties and filtration of clay suspensions for the factorial design used in static aging.]

\begin{tabular}{rccc}
\hline Fonte de variação & $\mathrm{VA}_{\mathrm{E}}(\mathrm{cP})$ & $\mathrm{VP}_{\mathrm{E}}(\mathrm{cP})$ & $\mathrm{VF}_{\mathrm{E}}(\mathrm{mL})$ \\
\hline Coeficiente de correlação $(\mathrm{R})$ & 0,989 & 0,987 & 0,986 \\
$\%$ de variaçãoexplicada* & 97,854 & 97,423 & 97,133 \\
$\mathrm{~F}_{\text {calculado }} / \mathrm{F}_{\text {tabelado }}$ & 9,030 & 7,486 & 6,708 \\
\hline Modelos matemáticos codificados para as suspensões argilosas \\
\hline $\mathrm{VA}_{\mathrm{E}}(\mathrm{cP})=18,93^{* *} \pm 1,28+\left(10,82 \mathrm{CA}^{* *} \pm 0,78\right)+\left(1,42 \mathrm{CA}^{2} \pm 0,93\right)+\left(3,27 \mathrm{TE}^{* *} \pm 0,78\right)-$ \\
$\left.\mathrm{VP}_{\mathrm{E}}(\mathrm{cP})=9,65^{* *} \pm 0,44\right)+\left(3,24 \mathrm{CA}^{* *} \pm 0,27\right)-\left(0,23 \mathrm{CA}^{2} \pm 0,32\right)+\left(1,73 \mathrm{TE}^{* *} \pm 0,27\right)-$ \\
$\mathrm{VF}_{\mathrm{E}}(\mathrm{mL})=23,77^{* *} \pm 0,93-\left(6,27 \mathrm{CA}^{* *} \pm 0,57\right)+(1,27 \mathrm{CA} \pm 0,67)+\left(3,69 \mathrm{TE}^{* *} \pm 0,57\right)+$ \\
$\left(0,58 \mathrm{TE}^{2} \pm 0,67\right)-(0,85 \mathrm{CA} \cdot \mathrm{TE} \pm 0,80)$
\end{tabular}

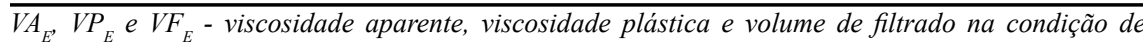
envelhecimento estático.

Tabela V - Análises de variância (ANOVA) e modelos matemáticos codificados das propriedades reológicas e de filtração das suspensões argilosas para o planejamento fatorial empregado sob envelhecimento dinâmico.

[Table V - Analysis of variance (ANOVA) and encoded mathematical models of the rheological properties and filtration of clay suspensions for the factorial design used in dynamic aging.]

\begin{tabular}{|c|c|c|c|}
\hline Fonte de variação & $V A_{D}(c P)$ & $V P_{D}(c P)$ & $V F_{D}(m L)$ \\
\hline Coeficiente de correlação (R) & 0,977 & 0,981 & 0,998 \\
\hline \% de variação explicada* & 95,439 & 96,195 & 99,629 \\
\hline $\mathrm{F}_{\text {calculado }} / \mathrm{F}_{\text {tabelado }}$ & 4,143 & 5,006 & 53,237 \\
\hline \multicolumn{4}{|c|}{ Modelos matemáticos codificados para as suspensões argilosas } \\
\hline \multicolumn{4}{|c|}{$\begin{array}{c}\mathrm{VA}_{\mathrm{D}}(\mathrm{cP})=26,67 * * \pm 3,07+\left(17,44 \mathrm{CA}^{* *} \pm 1,88\right)+\left(4,57 \mathrm{CA}^{2} \pm 2,23\right)+(1,01 \mathrm{TE} \pm 1,87)- \\
\left(6,49 \mathrm{TE}^{2 * *} \pm 2,22\right)-(0,63 \mathrm{CA} . \mathrm{TE} \pm 2,66)\end{array}$} \\
\hline \multicolumn{4}{|c|}{$\begin{array}{c}\left.\mathrm{VP}_{\mathrm{D}}(\mathrm{cP})=10,82 * * \pm 0,63\right)+(2,77 \mathrm{CA} * * \pm 0,39)-\left(1,81 \mathrm{CA}^{2 * *} \pm 0,46\right)+\left(1,82 \mathrm{TE}^{* *} \pm 0,39\right)- \\
\left(1,19 \mathrm{TE}^{2 * *} \pm 0,46\right)+\left(3,25 \mathrm{CA} \cdot \mathrm{TE}^{* *} \pm 0,55\right)\end{array}$} \\
\hline \multicolumn{4}{|c|}{$\begin{array}{c}\mathrm{VF}_{\mathrm{D}}(\mathrm{mL})=24,16^{* *} \pm 0,30-\left(5,45 \mathrm{CA}^{* *} \pm 0,18\right)+\left(1,23 \mathrm{CA}^{2 * *} \pm 0,22\right)+\left(3,80 \mathrm{TE}^{* *} \pm 0,18\right)+ \\
\left(0,83 \mathrm{TE}^{2} \pm 0,22\right)-(0,57 \mathrm{CA} \cdot \mathrm{TE} \pm 0,26)\end{array}$} \\
\hline
\end{tabular}

$V A_{D}, V P_{D}$ e $V F_{D}$ - viscosidade aparente, viscosidade plástica e volume de filtrado na condição de envelhecimento dinâmico.

e plástica e volume de filtrado indicaram que tanto a concentração de argila (CA) como a temperatura (TE) tem influência nas variáveis estudadas, sendo que as variáveis quadráticas e a interação entre as variáveis $\mathrm{CA}$ e TE para a $\mathrm{VP}_{\mathrm{E}}$ e $\mathrm{VF}_{\mathrm{E}}$ não foram estatisticamente significativas, ao nível de $95 \%$ de confiança. Para o envelhecimento dinâmico, o mesmo comportamento foi observado, ou seja, tanto a concentração de argila (CA) como a temperatura (TE) tem efeito nas propriedades avaliadas, sendo que a interação entre as variáveis CA e TE para a VA e para o VF não foi estatisticamente significativa, ao nível de $95 \%$ de confiança.

Nas Figs. 1 e 2 estão ilustradas as superfícies de resposta para as propriedades VA e VP, respectivamente.

Assim como já observado na Tabela III, a viscosidade aparente e a viscosidade plástica são fortemente influenciadas pelo aumento da concentração de argila e também pelo aumento da temperatura. Como mencionado, esse comportamento deve-se a maior intensidade das interações elétricas e de massa entre partículas predominantes com o aumento destas variáveis de entrada (CA e TE). Os altos 

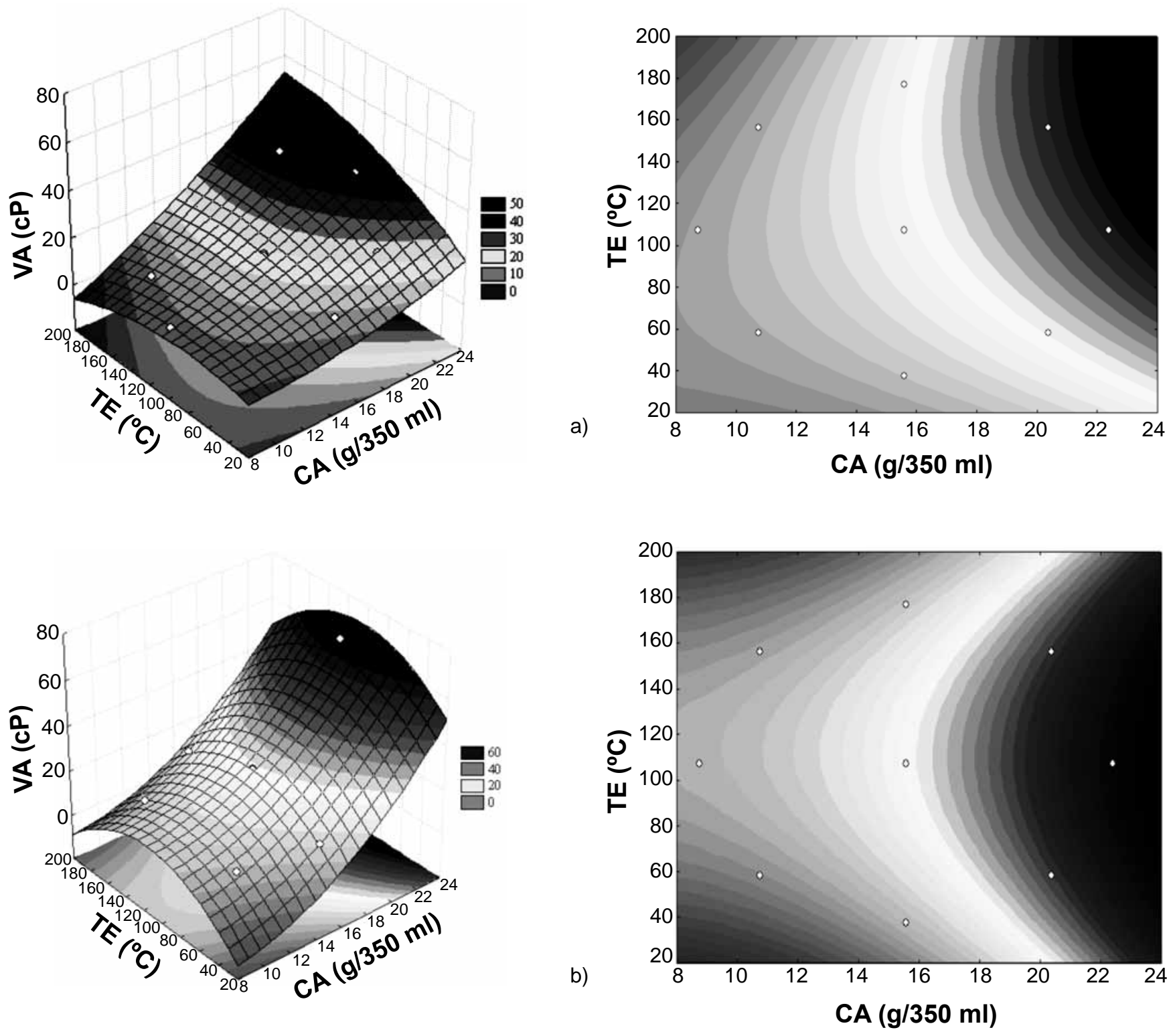

Figura 1: Superfícies de resposta para a viscosidade aparente (VA) sob condições de envelhecimento (a) estático e (b) dinâmico das suspensões argilosas.

[Figure 1: Response surfaces for the apparent viscosity under conditions of aging (a) static and (b) dynamic clay suspensions.]

valores de VA, obtidos principalmente para as suspensões submetidas ao envelhecimento nas temperaturas mais elevadas e com maiores concentrações de argila, caracterizam o estado de gelificação da suspensão. De acordo com as especificações da Petrobrás [16] para qualificação de argila, a viscosidade aparente deve apresentar valores superiores a $15,0 \mathrm{cP}$ e a viscosidade plástica, valores superiores a $4,0 \mathrm{cP}$. Assim, de acordo com a Fig. 1a, a melhor condição para viscosidade aparente encontra-se na faixa de concentação de argila entre $12 \mathrm{~g} / 350 \mathrm{~mL}$ a $16 \mathrm{~g} / 350 \mathrm{~mL}$. Para concentrações superiores a $19 \mathrm{~g} / 350 \mathrm{~mL}$ e $120^{\circ} \mathrm{C}$, as dispersões apresentam valores de viscosidade muito elevado, como consequência, maior grau de gelificação. Ainda de acordo com a Fig. 1 , as suspensões envelhecidas sob condições dinâmicas preparadas com baixas concentrações de argila e submetidas a temperaturas entre $100{ }^{\circ} \mathrm{C}$ a $140{ }^{\circ} \mathrm{C}$ apresentaram menores valores de VA e quando preparadas com altas concentrações de argila, maiores valores de VA (em comparação às amostras envelhecidas em condições estáticas). As suspensões submetidas ao envelhecimento dinâmico (Fig. $1 \mathrm{~b}$ apresentaram uma maior faixa de viscosidade aparente dentro das especificações da Petrobrás [16] do que as suspensões submetidas ao envelhecimento estático. De acordo com a Fig. 2, foi possível observar comportamento semelhante para os dois tipos de envelhecimento estudados; elevada temperatura associada à alta concentração de argila, resultando em maiores valores de VP. A partir da Fig. 2a, a melhor condição para $\mathrm{VP}_{\mathrm{E}}$ encontra-se na faixa de concentração de argila superior a $10 \mathrm{~g} / 350 \mathrm{~mL}$. As suspensões submetidas ao envelhecimento dinâmico 

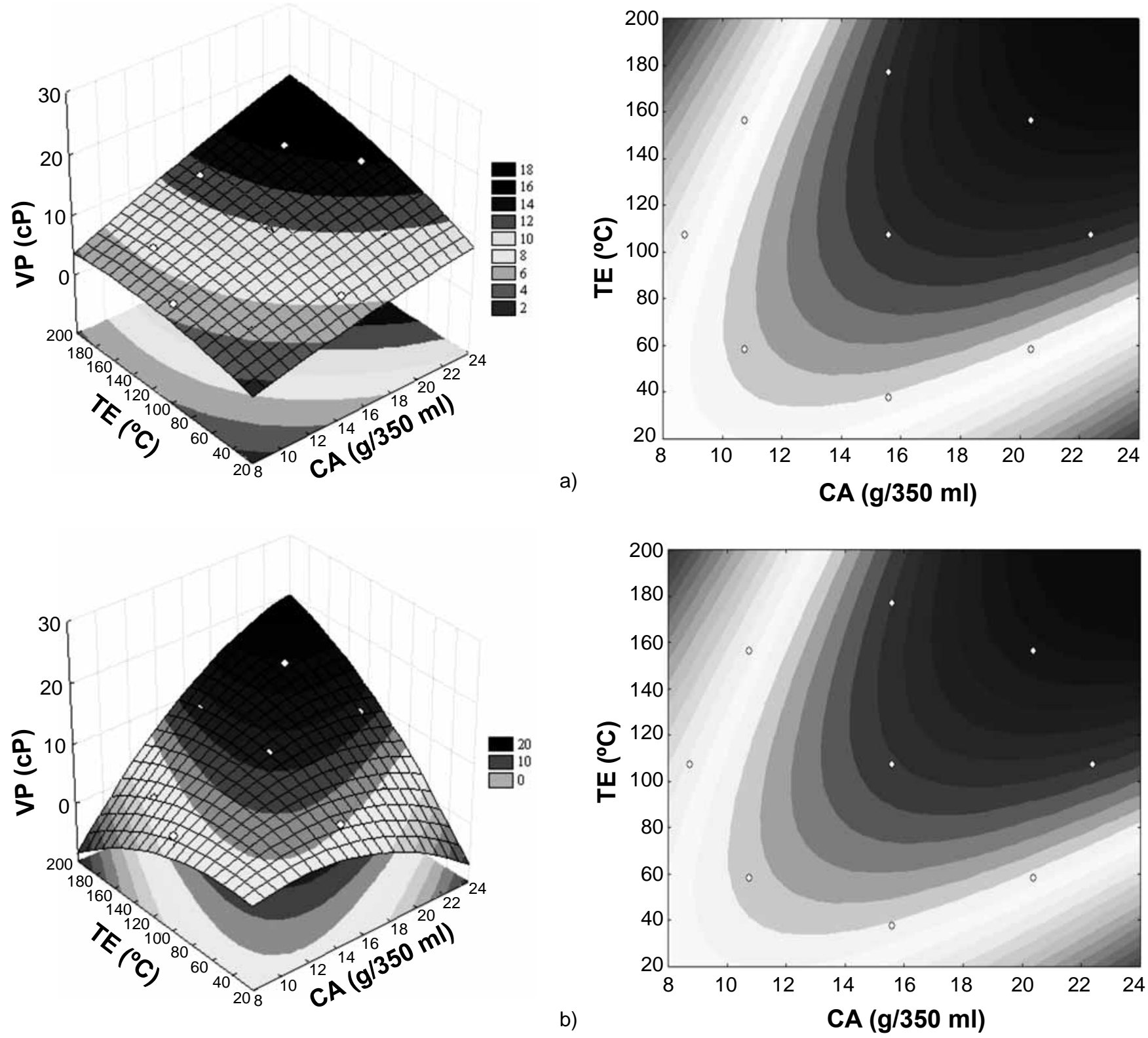

Figura 2: Superfícies de resposta para a viscosidade plástica (VP) sob condições de envelhecimento (a) estático e (b) dinâmico das suspensões argilosas.

[Figure 2: Response surfaces for the plastic viscosity under conditions of aging (a) static and (b) dynamic clay suspensions.]

apresentaram maiores valores de $\mathrm{VP}_{\mathrm{D}}$, conforme ilustrado na Fig. II (b).

Na Fig. 3 estão ilustradas as superfícies de resposta para o VF das suspensões estudadas.

Em relação ao comportamento obtido para o VF, observou-se que este diminui com o aumento da concentração de argila e aumenta, de maneira bastante expressiva, com o aumento da temperatura de envelhecimento a baixos teores de argila. Desta forma, ficou evidenciado que o aumento da temperatura, para as suspensões com baixas concentrações de argila, conduz os sistemas a maiores perdas por filtração. Observou-se ainda que as suspensões com elevada concentração de argila e baixa temperatura de envelhecimento apresentam valores de VF elevados, ou seja, mesmo as suspensões estando no estado de gelificação apresentam grandes volumes de filtrado, sugerindo que a água presente ou uma parcela da água presente encontrase livre e não presa nos reticulados conforme a teoria de Stefan [18]. Desta forma, pode-se inferir que os reticulados, formados pelas interações entre partículas de argila, retêm as moléculas de água dependendo do grau de floculação da dispersão, podendo-se ter géis com grandes quantidades de água livre, resultando em elevados volumes de filtrado e géis com menores quantidades de água livre, resultando em pequenos volumes de filtrado [19]. Este comportamento foi observado para os dois tipos de envelhecimento. No entanto, 

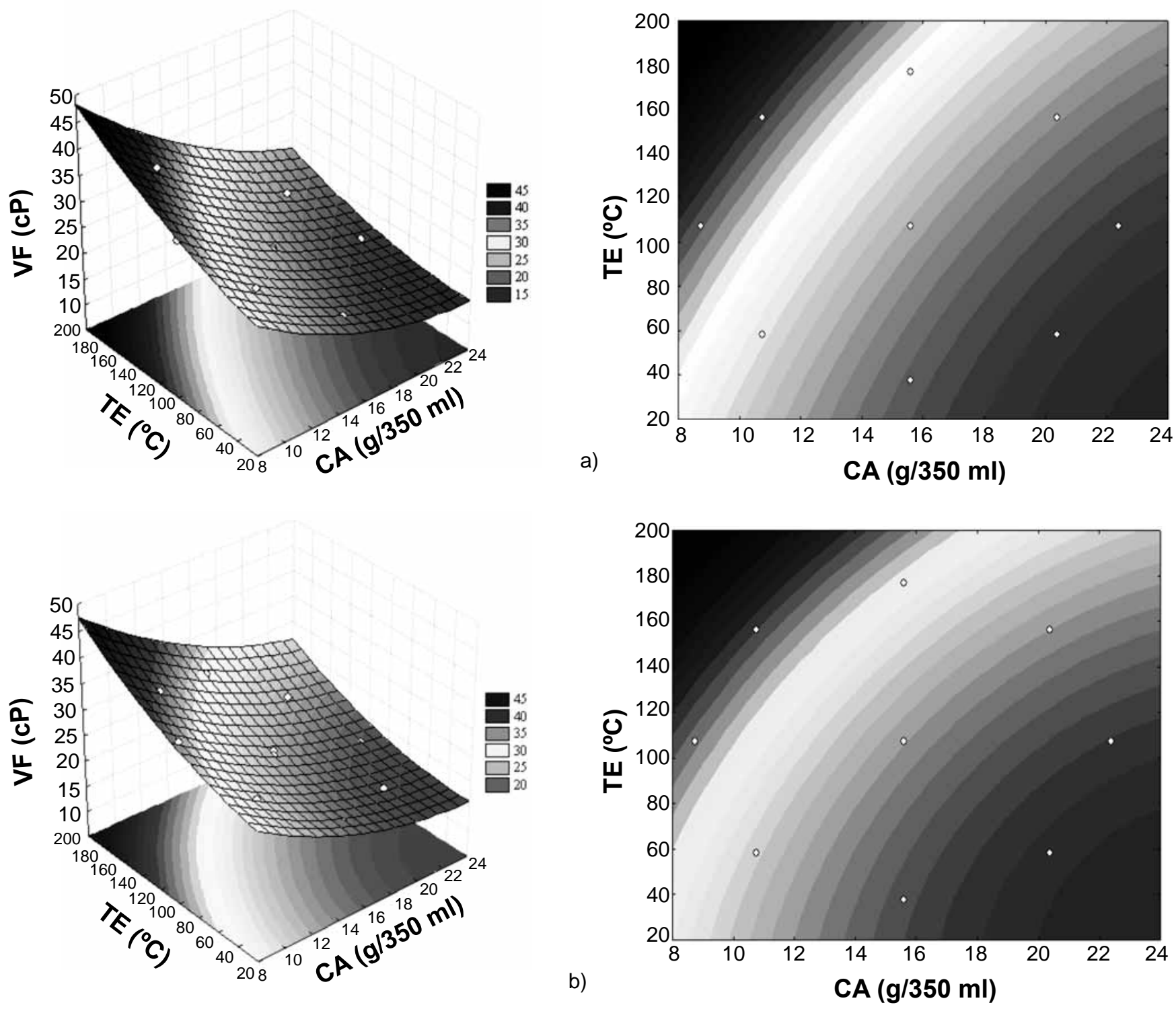

Figura 3: Superfícies de resposta para o volume de filtrado (VF) sob condições de envelhecimento (a) estático e (b) dinâmico das suspensões argilosas.

[Figure 3: Response surfaces for the volume of filtered under aging conditions (a) static and (b) dynamic clay suspensions.]

as suspensões submetidas ao envelhecimento dinâmico apresentaram uma maior faixa de valores inferiores à especificação da Petrobrás [16], quando comparado com as suspensões submetidas ao envelhecimento estático.

Três fatores são fundamentais para a variação das propriedades de filtração dos fluidos de perfuração submetidos a elevadas temperaturas [4]: a alteração da viscosidade do filtrado; o equilíbrio eletroquímico e a degradação química dos componentes do fluido. $\mathrm{O}$ aumento da temperatura promove uma redução da viscosidade do filtrado e, portanto, observa-se um aumento do volume de filtrado. Mudanças na temperatura também podem afetar o equilíbrio eletroquímico que regula o grau de floculação e agregação das suspensões, alterando a permeabilidade do reboco e, consequentemente, os volumes de filtrado. Por fim, muitos componentes presentes nos fluidos começam a degradar significativamente a temperaturas acima de $100{ }^{\circ} \mathrm{C}$; o aumento da taxa de degradação promovido pelo aumento da temperatura, também altera as propriedades de filtração.

\section{CONCLUSÕES}

Com o objetivo de avaliar as propriedades de suspensões argilosas sob diferentes condições térmicas, foi empregado um planejamento fatorial do tipo $2^{2} \mathrm{com}$ três experimentos no ponto central + configuração estrela. A análise estatística evidenciou que há relação entre as variáveis dependentes (VA, VP e VF) e as independentes (concentração de argila e temperatura de envelhecimento), bem como que os modelos matemáticos obtidos são estatisticamente significativos e preditivos nos dois tipos de envelhecimento térmico; maiores valores de VA e de VP 
são obtidos com o aumento na concentração de argila de $8,75 \mathrm{~g} / 350 \mathrm{~mL}$ a $22,4 \mathrm{~g} / 350 \mathrm{~mL}$, bem como com o aumento da temperatura de $38^{\circ} \mathrm{C} \mathrm{a} 176^{\circ} \mathrm{C}$. Este aumento decorre da maior intensidade das interações elétricas e de massa entre partículas, que se tornam predominantes com o aumento da concentração de argila e também da temperatura; suspensões com menores e, portanto, melhores valores de VF são obtidos com maiores concentrações de argila e menores temperaturas de envelhecimento; o aumento na temperatura de envelhecimento confere às suspensões maiores perdas por filtração, pois a temperatura afeta o equilíbrio eletroquímico que regula o grau de floculação e agregação das suspensões, conduzindo o fluido ao estado floculado-gel, com separação de fases apresentando água livre no sistema; as suspensões submetidas ao envelhecimento dinâmico apresentaram maiores valores de viscosidades aparente e plástica do que as suspensões submetidas ao envelhecimento estático, provavelmente devido a um maior grau de dispersão das particulas decorrente da taxa de cisalhamento aplicada; e nos dois tipos de envelhecimento (estático e dinâmico), o volume de filtrado comportou-se de maneira similar.

\section{AGRADECIMENTOS}

À Agência Nacional do Petróleo - ANP, ao Programa de Recursos Humanos PRH-25 e ao CNPq (Proc. 305729/20101) pelo apoio financeiro e concessão de bolsas, à Empresa Bentonit União Nordeste pelo fornecimento da argila bentonítica e ao LABDES pelo uso de suas instalações físicas.

\section{REFERÊNCIAS}

[1] P. Souza Santos, "Ciência e tecnologia das argilas", vol. 1, Ed. Edgard Blucher, S. Paulo, SP (1989).

[2] L. V. Amorim, Melhoria, Proteção e Recuperação da Reologia de Fluidos Hidroargilosos para Uso na Perfuração de Poços de Petróleo, Tese Dr., Engenharia de Processos, CCT/UFCG, Campina Grande, PB (2003).

[3] A. I. Ferraz, "Manual de Engenharia dos Fluidos de Perfuração", Div. Magcobar, Grupo Oilfield Prod. Dresser Ind. Inc., Houston, Texas, EUA (1977).

[4] H. C. H. Darley, G. R. Gray, "Composition and properties of drilling and completion fluids", $5^{\text {th }}$ Ed., Gulf Publ., Houston, Texas, EUA (1988).
[5] J. E. Thomas, "Fundamentos de engenharia de petróleo", Ed. Interciência, Rio de Janeiro, RJ (2001).

[6] R. K Clark, Impact of environmental regulations on drilling fluid technology, J. Pet. Techn. 46, 9 (1994) 804809.

[7] J. Kelly Jr., D. E. Hawk, Mud Additives in Deep Wells, Oil Gas J. 2 (1961) 145-155.

[8] M. R. Annis, High Temperature Properties of WaterBase Drilling Fluids, J. Petrol. Technol., Trans. AIME 240 (1967) 1074-1080.

[9] K. H. Hiller, Rheological Measurements of Clay Suspensions at high temperatures and pressures, J. Petrol. Technol. 17 (1963) 779-789.

[10] P. M. Singh, V. P. Sharma, Effect of additives and aging on the rheological properties of water based drilling fluids, Energy Sources 13 (1991) 369-387.

[11] B. J. Briscoe, P. F. Luckham, S. R. Ren, The properties of drilling muds at high-pressures and high-temperatures, Phil. Trans. Royal Soc. London, Series A, 348 (1994) 179207.

[12] P. F. Luckham, S. Rossi, The colloidal and rheological properties of bentonite suspensions, Adv. Colloid Interf. Sci. 82 (1999) 43-92.

[13] E. Santoyo, S. Santoyo-Gutierrez, A. Garcia, G. Espinosa, S. I. Moya, Rheological property measurement of drilling fluids used in geothermal wells, Appl. Thermal Eng. 21 (2001) 283-302.

[14] B. Barros Neto, I. S. Scarminio, R. E. Bruns, Planejamento e otimização de experimentos, Ed. UNICAMP, S. Paulo, SP (1996).

[15] Statsoft, Inc. Statistica for Windows, version 7.0 (2000). [16] Petrobrás, Viscosificante para fluidos usados na exploração e produção de poços de petróleo e gás, EP-1EP00011-A (2011).

[17] M. M. Knechtel, S. H. Patterson, Bentonite Deposits in Marine Cretaceous Formations, Hardin District, Montana and Wyoming, U.S. Geol. Survey Bull. 1023 (1956) Drilling Fluids Components 605.

[18] P. Stefan, Métodos de ensaio de argilas e bentonitas para fluidos de perfuração, Cerâmica 12, 47-48 (1966) 266.

[19] C. A. Leal, R. C. A. M. Nascimento, L. V. Amorim, Estudo das propriedades de fluidos de perfuração hidroargilosos após envelhecimento térmico, $\mathrm{X}$ Congresso Ibero-Americano em Engenharia Mecânica, Porto, Portugal (2011)

(Rec. 28/02/2012, Rev. 10/06/2012, Ac. 26/06/2012) 